\title{
Performance of High-Level Reconstruction at Belle II
}

\author{
Francesco Tenchini ${ }^{*}$ \\ Deutsches Elektronen-Synchrotron (DESY) \\ E-mail: francesco.tenchini@desy.de
}

Belle II is a new $B$ factory located at the SuperKEKB electron-positron collider in Tsukuba, Japan, which started operation with the full detector configuration in spring 2019. During operation in 2019 it collected $10.5 \mathrm{fb}^{-1}$ of collision data. These proceedings present early results and a discussion of the performance of the high-level reconstruction of events for analysis.

18th International Conference on B-Physics at Frontier Machines - Beauty2019-

29 September / 4 October, 2019

Ljubljana, Slovenia

* Speaker.

${ }^{\dagger}$ On behalf of the Belle II collaboration. 


\section{Introduction}

Belle II is a second generation high-luminosity $B$ factory which represents a major upgrade of the previous Belle experiment. It is coupled to the SuperKEKB accelerator (Tsukuba, Japan) an energy asymmetric electron-positron collider designed to reach an unprecedented instantaneous luminosity of $8.0 \times 10^{35} \mathrm{~cm}^{-2} \mathrm{~s}^{-1}$. Over its lifetime, the Belle II experiment aims to record $50 \mathrm{ab}^{-1}$ of data, a factor of 50 more than the Belle experiment, enabling an extensive physics program [1]. During operation in 2019, Belle II collected data for the first time with almost the full detector configuration ("Phase III") and recorded approximately $10.5 \mathrm{fb}^{-1}$ of collision data, allowing early performance studies and "rediscovery" of known physics processes.

The remainder of this contribution is organized as follows. Highlights of the high-level reconstruction are presented in Sections 2-5: the resolution of the beam spot, the determination of the $D^{0}$ lifetime, charged particle identification (PID) and full-event interpretation (FEI). Section 6 gives a summary.

It must be noted that the results shown in these proceedings were produced at different times during the year and with the data available at the time. Therefore, most of them are derived from a substantially smaller dataset than the full $10.5 \mathrm{fb}^{-1}$.

\section{Beam Spot Resolution}

The key to achieving the luminosity goal of Belle II lies in its novel nanobeam scheme, which is designed to achieve an extremely small beam spot [2]. At the time of this report a value of $\beta_{y}^{*}=2 \mathrm{~mm}$ has been achieved, with an ultimate goal of $\beta_{y}^{*}=300 \mu \mathrm{m}$. Achieving a sufficient resolution to take advantage of such a small beam spot is possible thanks to the state-of-the-art Belle II tracking system, composed of two layers of silicon pixel detector (PXD), four layers of silicon strip detector (SVD) and a drift chamber (CDC).

It is possible to exploit low track multiplicity events to accurately measure the impact parameter resolution in the interaction region. To this end, we select events containing two back-to-back tracks of opposite charge, with high transverse momentum $\left(p_{T}>1 \mathrm{GeV}\right)$ and associated hits in all three tracking subdetectors. The width of the transverse impact parameter $d_{0}$ is then estimated by taking half of the symmetric range around the median containing $68 \%$ of the $d_{0}$ distribution, $\sigma_{68}\left(\Delta d_{0}\right)$. If we assume both tracks originate from a common vertex, then $1 / \sqrt{2}$ of the width of $\Delta d_{0}=\left(d_{0}(+)-d_{0}(-)\right)$ is a good estimator of the $d_{0}$ resolution. Using an early subset of Phase 3 data we measure $\sigma_{68}\left(\Delta d_{0}\right) / \sqrt{2}=14.1 \pm 0.1 \mu \mathrm{m}$, which is approximately a factor of two better than Belle (Fig. 1).

\section{3. $D^{0}$ Lifetime}

The excellent vertexing performance enabled the first measurement of the $D^{0}$ lifetime, performed by reconstructing $D^{*+} \rightarrow D^{0}\left(K^{-} \pi^{+}\right) \pi^{+}$events on an early sample corresponding to an integrated luminosity of $0.34 \mathrm{fb}^{-1}$. To this end, we select tracks originating from the interaction region $\left(\left|d_{0}\right|<1 \mathrm{~cm},\left|z_{0}\right|<3 \mathrm{~cm}\right)$, which pass an invariant-mass selection around the nominal $D^{0}$ mass value $(1.80<m(K \pi)<1.95 \mathrm{GeV})$. To reject $D^{*+}$ originating from $B$ decays we require a 

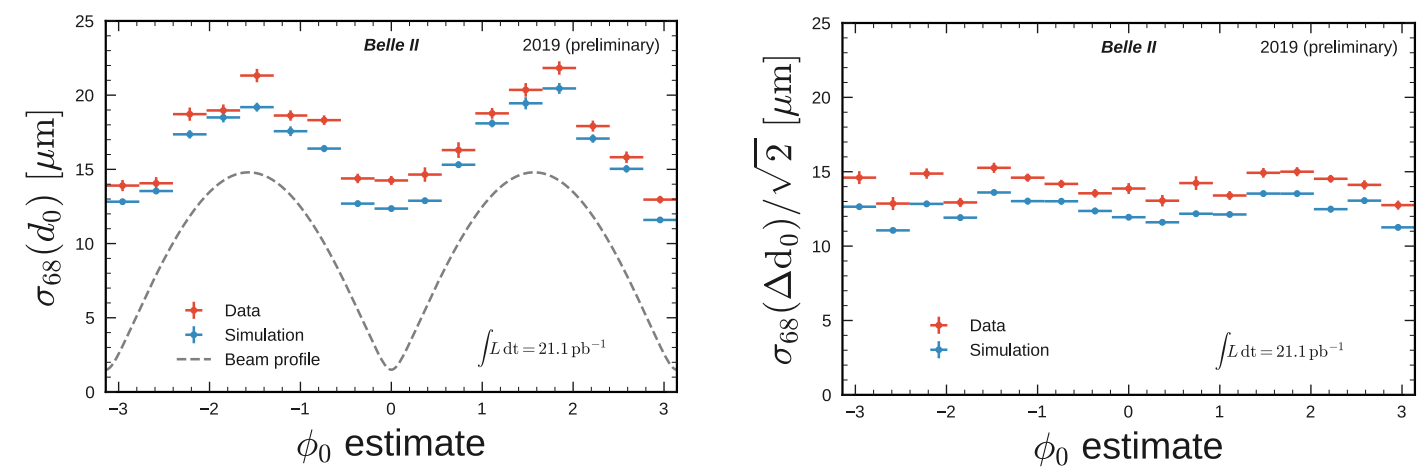

Figure 1: Left: Width of the transverse impact parameter $d_{0}$ distribution as a function of the azimuthal angle $\phi_{0}$. Results from data (red) and simulation (blue) are overlaid with the beam profile shape used in simulation (dashed grey). The difference from the latter is attributed to detector resolution. Right: Resolution of the transverse impact parameter as a function of the azimuthal angle in data and simulation [3].

center-of-mass momentum of the $D^{*+}$ greater than $2.5 \mathrm{GeV}$. We then apply a simultaneous vertex fit to the full decay tree [7] to extract the proper decay time of the $D^{0}$. As part of the fit, we constrain the $D^{*+}$ vertex to lie inside the measured interaction region and $m(K \pi)$ to be the mass of the $D^{0}$. Finally, we select the signal region $5.346<Q<6.353 \mathrm{MeV}, 1.848<m(K \pi)<1.879 \mathrm{GeV}$, where $Q=m\left(D^{*+}\right)-m\left(D^{0}\right)-m\left(\pi^{+}\right)$and $m(K \pi)$ is taken before the mass-constrained fit. The proper decay time distribution is fit to the sum of two Gaussian functions, each convolved with an exponential (Fig. 2). We extract $\tau=370 \pm 40$ (stat) fs, which is in agreement with existing measurements [5].

\section{Particle Identification}

Particle identification (PID) is an important tool to isolate physics signals of interest from events with the same topology and fake candidates originating from combinatorial background. In Belle II all subdetectors contribute to charged PID; each particle candidate is assigned a likelihood $\mathscr{L}_{i}$, where $i \in[e, \mu, K, p, d]$, which is used for particle separation. Figure 3 shows an example of PID performance in the $D^{*+} \rightarrow D^{0}\left(K^{-} \pi^{+}\right) \pi^{+}$channel, where the slow pion is used to tag the meson charges, which provides an alternate means of identifying the $K$ or $\pi$. The $K / \pi$ separation can then be quantified as a function of the binary likelihood ratio

$$
R_{K / \pi}=\frac{\mathscr{L}_{K}}{\mathscr{L}_{K}+\mathscr{L}_{\pi}}
$$

where the dominant contribution to the likelihoods come from the time-of-propagation (TOP) and ring-imaging Cherenkov (ARICH) detectors, as well as energy loss in the drift chamber (CDC) for low momentum tracks.

\section{Full Event Interpretation}

The full reconstruction of the second $B$ meson is a fundamental tool to study missing-energy 


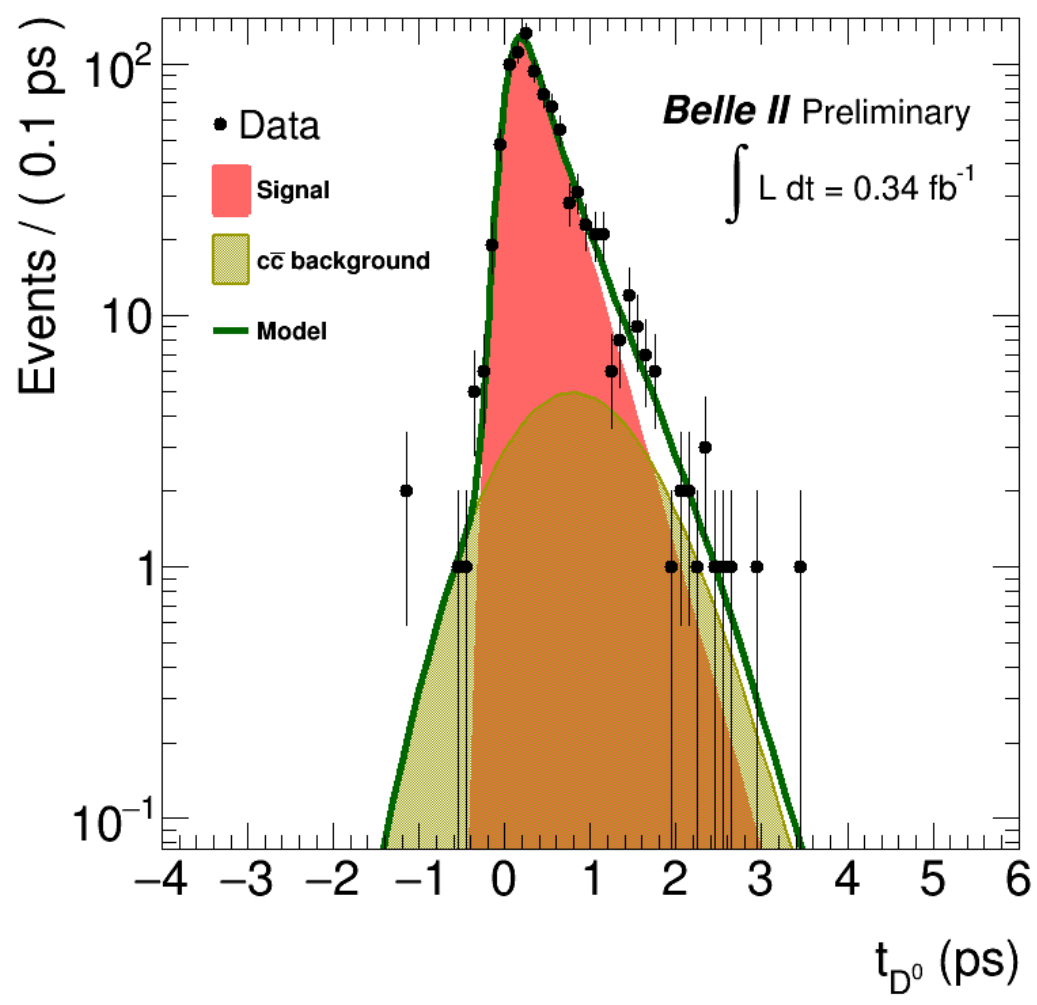

Figure 2: Fit to the measured proper decay time of the $D^{0}$ candidates. The fit functions represent correctly reconstructed $D^{*+}$ mesons and misrecontructed $c \bar{c}$ background [4].
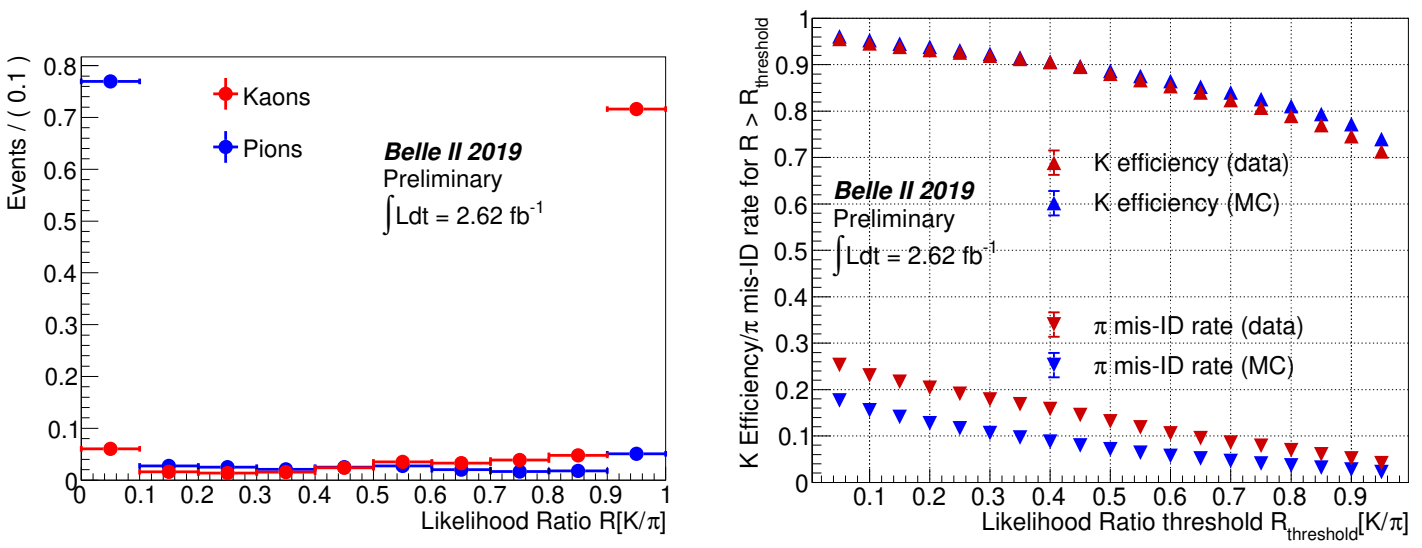

Figure 3: Left: Likelihood ratio distribution for $K$ and $\pi$. Right: $K / \pi$ separation in data and Monte Carlo (MC) simulation as a function of the $R_{K / \pi}$ threshold used in the selection. The increased mis-identification rate is attributed to limited understanding of the PID detectors' features during early running. [6]. 


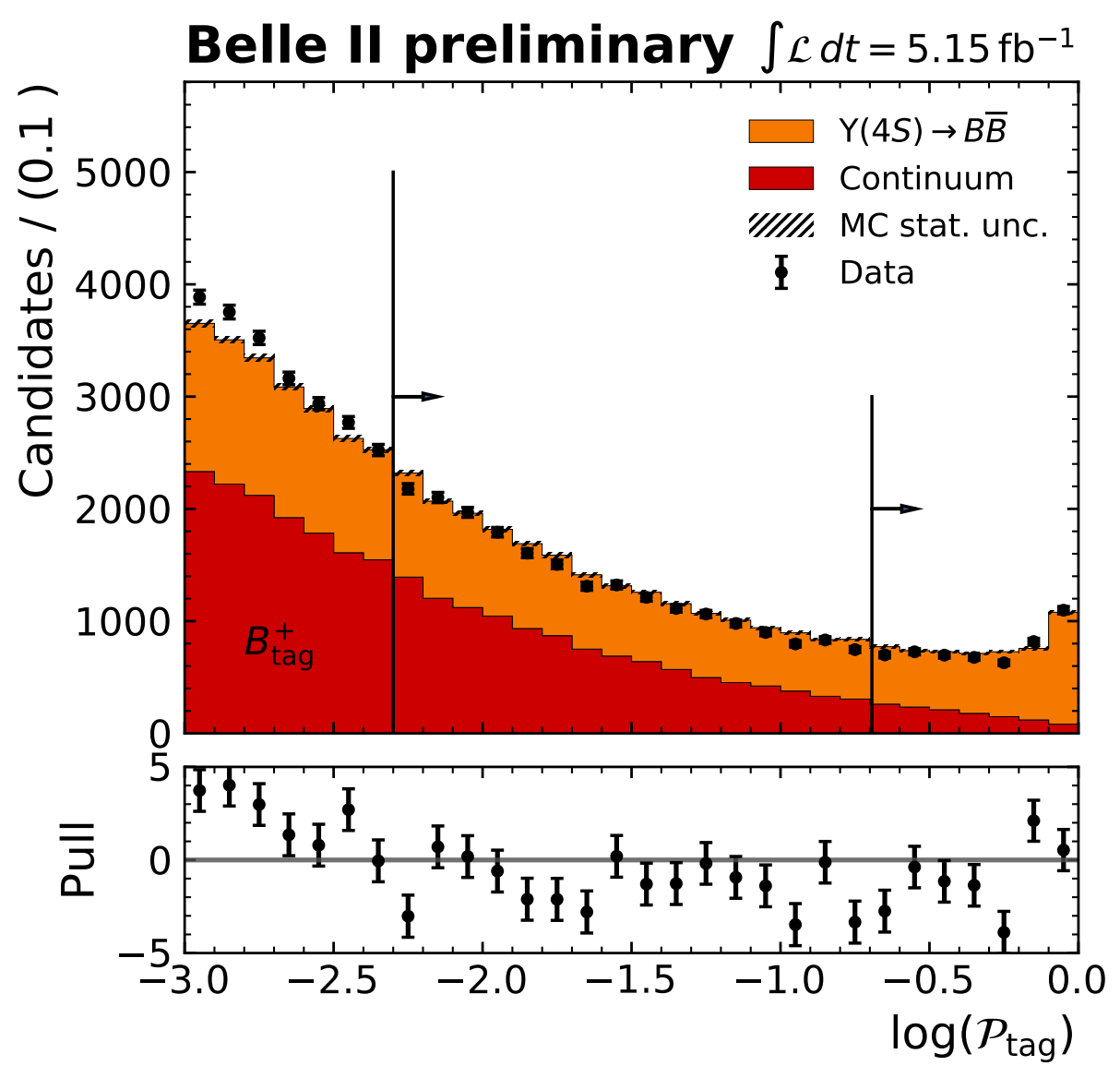

Figure 4: Comparison of the distribution of the FEI classifier output $\mathscr{P}_{\text {tag }}$ in early Phase III data to the expected shape from simulation. Two possible selection criteria are shown, corresponding to $\mathscr{P}_{\text {tag }}>0.1$ and $\mathscr{P}_{\text {tag }}>0.5[9]$.

decays at $B$ factories. This method allows the inference of the kinematics of the first meson (signal) from the second (tag) in cases where the signal $B$ decays into states containing one or more undetected particles.

Exclusive-tag reconstruction in Belle II makes use of a new algorithm called Full Event Interpretation (FEI) [8], a multi-stage classifier which reconstructs $B$ meson candidates by combining long-lived particle candidates (e, $\left.\mu, K, \pi, K_{L}, \gamma\right)$ into $\mathrm{O}(100)$ possible channels. At each stage the particles are combined to form composites $\left(J / \Psi, \pi^{0}, K_{S}, D, D^{*}\right)$ to which a probability is assigned in the form of a multivariate classifier, previously trained on simulated events. To evaluate the FEI performance in data we consider the $B^{-} \rightarrow X e^{-} v$ decay channel, where $X$ is an inclusive hadron system, and apply the following loose preselection criteria to suppress continuum events:

- $-0.15<\Delta E<0.10 \mathrm{GeV}$

- $\cos \left(\theta_{\text {thrust }}\right)<0.95$. 
Here $\Delta E$ is the difference between the tag $B$ energy and half the beam energy, and $\theta_{\text {thrust }}$ is the angle between the thrust axis of the $\operatorname{tag} B$ and that of the remainder of the event. For each event, only the $B$ candidate with the highest FEI probability $\mathscr{P}_{\text {tag }}$ is kept. The resulting probability distribution is displayed in Fig. 4.

A selection on the classifier output is helpful in suppressing incorrectly reconstructed $B$ meson candidates, including fakes originating from continuum background. The total signal yield can then be extracted by a fit to the beam-constrained mass, $m_{\mathrm{bc}}=\sqrt{s / 4-\mathbf{p}_{\mathbf{B}}{ }^{2}}$ where $s$ is the squared total energy of the center of mass system (CMS) and $\mathbf{p}_{\mathbf{B}}$ is the momentum vector of the $\operatorname{tag} B$ in the CMS. This is shown in Fig. 5.

The distributions of squared missing mass $\left(m_{\text {miss }}^{2}\right)$ and lepton momentum spectrum in the signal $B$ meson rest frame $\left(p_{e}^{*}\right)$ are shown in Fig. 6. Data are compared to the shapes expected from simulation and are found to be in good agreement.
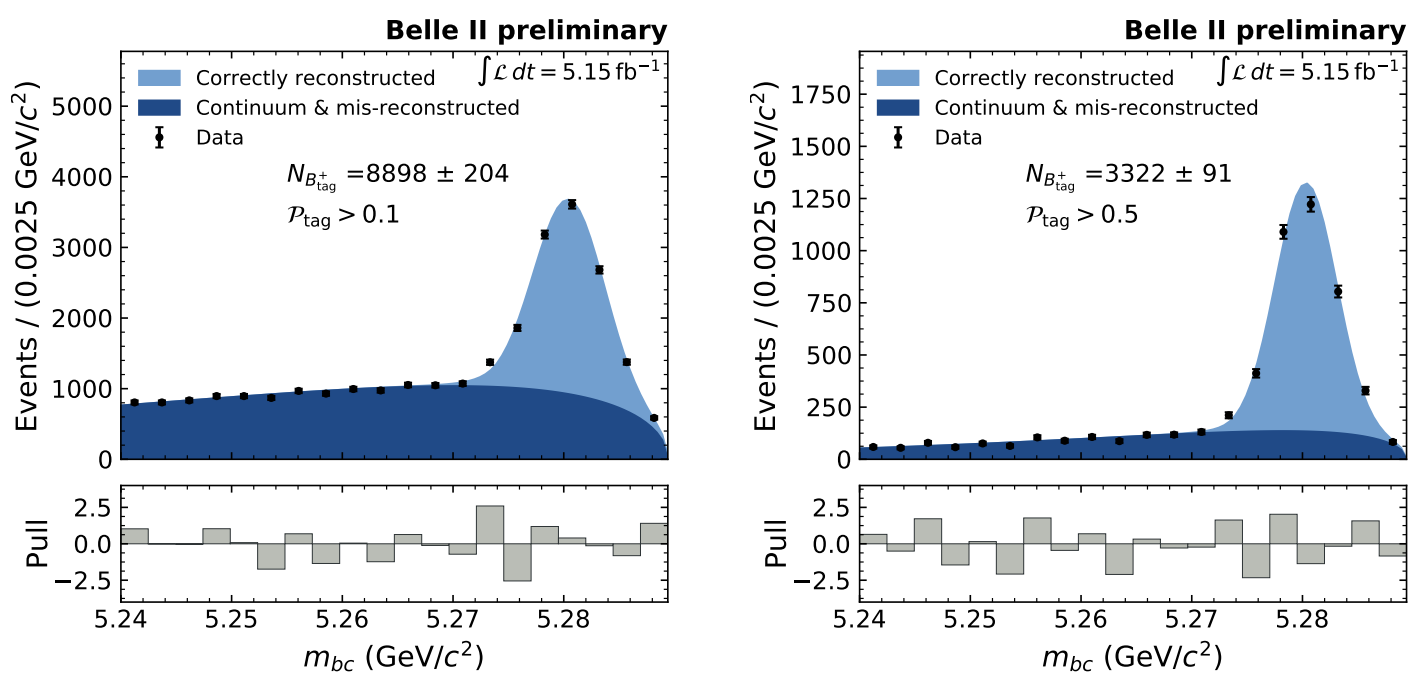

Figure 5: Fit to the beam-constrained mass of $\operatorname{tag} B$ candidates for two different $\mathscr{P}_{\text {tag }}$ selections. Wellreconstructed $B$ mesons are modeled by a Crystal Ball distribution, while an ARGUS shape is used to model the background [9].

\section{Summary}

During 2019 the Belle II experiment collected its first $10.5 \mathrm{fb}^{-1}$ of collision data in a nearfull detector configuration. Early Belle II measurements validate the detector readiness for physics analysis. They show a good performance in track and vertex reconstruction, ongoing improvements in particle identification as well as promising results in $B$ meson full reconstruction. 

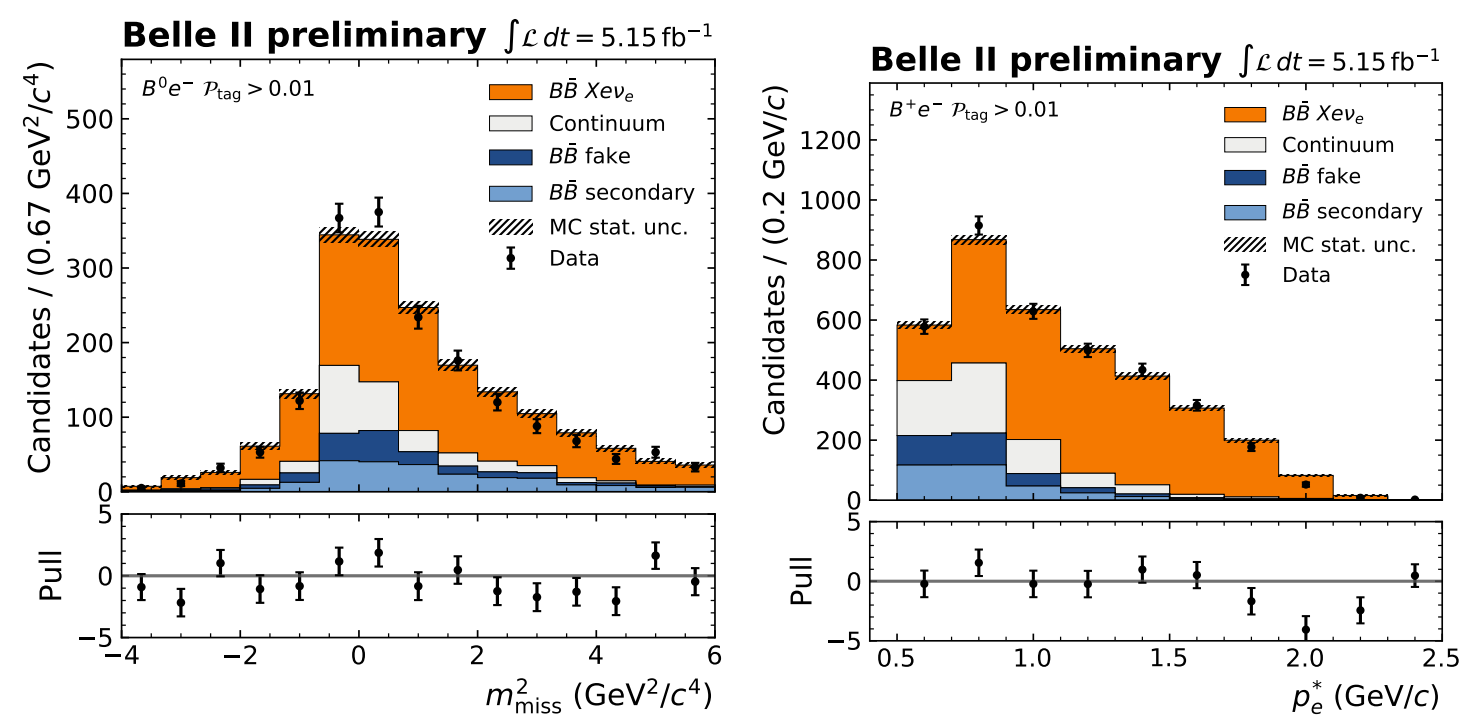

Figure 6: Left: Missing-mass distribution of inclusively reconstructed $B^{-} \rightarrow X e^{-} v$ decays combined with a $B^{+}$meson reconstructed via hadronic channels using FEI. Right: Momentum spectrum of the electron in the rest frame of the $B^{-}$, which is reconstructed from the $\operatorname{tag} B$ kinematics [9].

\section{References}

[1] E. Kou, P. Urquijo et al. (Belle II collaboration), "The Belle II Physics Book", PTEP 2019 (2019) no.12, 123C01, arXiv:1808.10567 [hep-ex].

[2] K. Akai et al. (SuperKEKB collaboration), "SuperKEKB Collider", Nucl. Instru. Meth. A907, 188 (2018), arXiv:1809.01958 [physics.acc-ph].

[3] N. Braun et al., "Study of the impact parameter resolution andthe beam profile in early Phase 3 data", https://docs.belle2.org/record/1536/files/BELLE2-NOTE-PL-2019-011.pdf .

[4] G. de Marino and G. Casarosa, " $D^{0}$ Lifetime On Bucket6 Phase3 Data", https://docs.belle2.org/record/1512/files/BELLE2-NOTE-PL-2019-003.pdf .

[5] M. Tanabashi et al., (Particle Data Group), Phys. Rev. D 98, 030001

[6] S. Sandilya and A. Schwartz, "Kaon and Pion Identification Performances in Phase III data", https://docs.belle2.org/record/1595/files/BELLE2-NOTE-PL-2019-022.pdf .

[7] J.-F. Krohn et al., "Global Decay Chain Vertex Fitting at B-Factories", arXiv:1901.11198 [hep-ex].

[8] T. Keck et al., "The Full Event Interpretation - An exclusive tagging algorithm for the Belle II experiment", Comput. Softw. Big Sci. 3, 6 (2019), arXiv:1807.08680 [hep-ex].

[9] W. Sutcliffe and F. Bernlochner, "Full Event Interpretation Reconstruction Performance with 5.15 $\mathrm{fb}^{-1}$ of Phase III data in proc 9 and bucket 7", https://docs.belle2.org/record/1712/files/BELLE2-NOTE-PL-2019-030.pdf . 